\title{
Mass Measurements of Neutron-Deficient Yb Isotopes and Nuclear Structure at the Extreme Proton-Rich Side of the $N=82$ Shell
}

\author{
Sönke Beck®, ${ }^{1,2,{ }^{*}}$ Brian Kootte, ${ }^{3,4}$ Irene Dedes, ${ }^{5,6}$ Timo Dickel, ${ }^{1,2}$ A. A. Kwiatkowski, ${ }^{3,7}$
} Eleni Marina Lykiardopoulou, ${ }^{8,3}$ Wolfgang R. Plaß, ${ }^{1,2}$ Moritz P. Reiter, ${ }^{1,3,9}$ Corina Andreoiu, ${ }^{10}$ Julian Bergmann, ${ }^{1}$ Thomas Brunner, ${ }^{11}$ Dominique Curien, ${ }^{12}$ Jens Dilling, ${ }^{3,8}$ Jerzy Dudek, ${ }^{12,6}$ Eleanor Dunling, ${ }^{3,13}$ Jake Flowerdew, ${ }^{14}$ Abdelghafar Gaamouci, ${ }_{15}^{15}$ Leigh Graham, ${ }^{3}$ Gerald Gwinner, ${ }^{4}$ Andrew Jacobs, ${ }^{8,3}$ Renee Klawitter, ${ }^{3}$ Yang Lan, ${ }^{8}$ Erich Leistenschneider, ${ }^{8,3}$ Nikolay Minkov, ${ }^{16}$ Victor Monier, ${ }^{3}$ Ish Mukul, ${ }^{3}$ Stefan F. Paul, ${ }^{3}$ Christoph Scheidenberger, ${ }^{1,2,17}$ Robert I. Thompson, ${ }^{14}$ James L. Tracy, Jr., ${ }^{3}$ Michael Vansteenkiste, ${ }^{3}$ Hua-Lei Wang, ${ }^{18}$ Michael E. Wieser, ${ }^{14}$ Christian Will, ${ }^{1}$ and Jie Yang ${ }^{6,18}$

${ }^{1}$ II. Physikalisches Institut, Justus-Liebig-Universität, 35392 Gießen, Germany

${ }^{2}$ GSI Helmholtzzentrum für Schwerionenforschung GmbH, 64291 Darmstadt, Germany

${ }^{3}$ TRIUMF, Vancouver, British Columbia V6T 2A3, Canada

${ }^{4}$ Department of Physics and Astronomy, University of Manitoba, Winnipeg, Manitoba R3T 2N2, Canada

${ }^{5}$ Institute of Nuclear Physics, Polish Academy of Sciences, PL-31 342 Kraków, Poland

${ }_{6}^{6}$ Institute of Physics, Marie Curie-Skłodowska University, PL-20 031 Lublin, Poland

${ }^{7}$ Department of Physics and Astronomy, University of Victoria, Victoria, British Columbia V8P 5C2, Canada

${ }^{8}$ Department of Physics and Astronomy, University of British Columbia, Vancouver, British Columbia V6T 1Z1, Canada

${ }^{9}$ School of Physics and Astronomy, University of Edinburgh, Edinburgh EH9 3FD, Scotland, United Kingdom

${ }^{10}$ Department of Chemistry, Simon Fraser University, Burnaby, British Columbia V5A 1S6, Canada

${ }^{11}$ Physics Department, McGill University, H3A 2 T8 Montréal, Québec, Canada

${ }^{12}$ Université de Strasbourg, CNRS, IPHC UMR 7178, F-67 000 Strasbourg, France

${ }^{13}$ Department of Physics, University of York, York YO10 5DD, United Kingdom

${ }^{14}$ Department of Physics and Astronomy, University of Calgary, Calgary, Alberta T2N 1N4, Canada

${ }^{15}$ Faculté de Physique, University of Science and Technology Houari Boumediene, BP 32, El Alia, 16111 Bab Ezzouar, Algiers, Algeria

${ }^{16}$ Institute of Nuclear Research and Nuclear Energy, Bulgarian Academy of Sciences, BG-1784 Sofia, Bulgaria

${ }^{17}$ Helmholtz Forschungsakademie Hessen für FAIR (HFHF), GSI Helmholtzzentrum für Schwerionenforschung, Campus Gießen, 35392 Gießen, Germany

${ }^{18}$ School of Physics and Microelectronics, Zhengzhou University, Zhengzhou 450001, China

(Received 13 April 2021; revised 30 June 2021; accepted 21 July 2021; published 10 September 2021)

High-accuracy mass measurements of neutron-deficient $\mathrm{Yb}$ isotopes have been performed at TRIUMF using TITAN's multiple-reflection time-of-flight mass spectrometer (MR-TOF-MS). For the first time, an MR-TOF-MS was used on line simultaneously as an isobar separator and as a mass spectrometer, extending the measurements to two isotopes further away from stability than otherwise possible. The ground state masses of ${ }^{150,153} \mathrm{Yb}$ and the excitation energy of ${ }^{151} \mathrm{Yb}^{m}$ were measured for the first time. As a result, the persistence of the $N=82$ shell with almost unmodified shell gap energies is established up to the proton drip line. Furthermore, the puzzling systematics of the $h_{11 / 2}$-excited isomeric states of the $N=81$ isotones are unraveled using state-of-the-art mean field calculations.

DOI: 10.1103/PhysRevLett.127.112501

Experimental and theoretical studies of exotic nuclei, i.e., very short-lived nuclei far away from the valley of stability in the chart of the nuclides, present a unique and important way to gain a general understanding of the

Published by the American Physical Society under the terms of the Creative Commons Attribution 4.0 International license. Further distribution of this work must maintain attribution to the author(s) and the published article's title, journal citation, and DOI. atomic nucleus and the governing interactions of its constituents. Exotic nuclei reveal novel properties, unknown in more stable nuclei, such as nuclear halos and skins, and exotic decay modes [1,2]. A deeper understanding of nuclear structure hinges on theoretical models. Extending experimental data toward the drip lines is decisive for testing prediction capacities of theories, estimating the model uncertainties and thus for improving models and theories [3].

One striking effect, which may occur in exotic nuclei, is a change in the nuclear shell structure toward the proton or 
neutron drip lines; shells can weaken or disappear, and new magic numbers appear [4,5]. On the neutron-rich side of the nuclear chart, shell closures have been shown to vanish far from stability for the neutron numbers $N=20$ and $N=28$ [6,7], and new shell closures have been found for $N=32$ and $N=34$ [8-15]. The $N=82$ shell closure has been studied for neutron-rich nuclei down to Cd [16-18]. The data in the neutron-deficient region are incomplete, and the evolution of the $N=82$ shell toward the proton drip line is not known. In this Letter, the $N=82$ shell closure is investigated by mass measurements up to the proton drip line.

Series of nuclear isomers are known to occur near shell closures. A unique sequence of isomers exists in the $N=81$ isotones with even $Z$, ranging from ${ }^{131} \mathrm{Sn}$ to ${ }^{149} \mathrm{Er}[19,20]$. This sequence is remarkable, because the excitation energies of these $J^{\pi}=11 / 2^{-}$isomers stay approximately constant at $750 \mathrm{keV}$ between ${ }^{139} \mathrm{Ce}$ and ${ }^{149} \mathrm{Er}$ [21-25], over a range of eleven isotones. Such an effect is unique for isomers throughout the chart of the nuclides, and its origin has been considered enigmatic since its discovery more than 60 years ago $[22,25,26]$. The dependence of energy vs total angular momentum within noncollective excitation regimes is usually strongly irregular according to nuclear mean-field theory [27]. Yet here, the experimental data of the series are extended, and the origin of the constant excitation energies is explained using state-of-the-art mean-field calculations.

A major challenge for experiments with exotic nuclei at radioactive ion beam facilities is isobaric contamination. Nuclei closer to stability and molecules are usually produced with rates many orders of magnitude higher than those of the nuclei of interest and hamper measurements of the exotic nuclei. Recently, multiple-reflection time-offlight mass spectrometers (MR-TOF-MS) [28,29] have been established as isobar separators $[30,31]$ and even isomer separators [32]. They feature very high mass separation powers of several $10^{5}$ and short cycle times, enabling access to very short-lived $(\approx \mathrm{ms})$ nuclides and high ion rates $\left(10^{6}\right.$ ions/s). MR-TOF-MS can also be used for direct mass measurements of exotic nuclei $[9,12,33,34]$ and diagnostic purposes [35-37]. In an MR-TOF-MS, ions are cooled in a radio-frequency (RF) ion trap (injection trap), injected into a time-of-flight (TOF) analyzer, in which the ions are stored between two ion reflectors and dispersed in TOF according to their mass-to-charge ratios. Mass separation is then achieved by the subsequent removal of the unwanted ions using a fast-switching deflector, such as a Bradbury-Nielsen gate [30], a pulsed drift tube [38], or one of the reflectors [39]. A novel method for mass separation in an MR-TOF-MS is the dynamical retrapping of the ions in the injection trap after the TOF dispersion procedure [40]. This retrapping technique is highly mass selective; the ions of interest can be stored, while other ions are removed. In contrast with the other methods, it allows an
MR-TOF-MS to act as an isobar separator for its own mass measurements. It has been developed for the MR-TOF-MS $[41,42]$ at TRIUMF's Ion Trap for Atomic and Nuclear Science (TITAN) [43], but it could also be used to add one or several stages of isobar separation to mass measurements in other MR-TOF-MS worldwide.

In this Letter, mass-selective retrapping has been employed for the first time online. High-accuracy mass measurements of neutron-deficient $\mathrm{Yb}$ isotopes were performed using TITAN's MR-TOF-MS. The nuclei were produced in spallation reactions at the Isotope Separator and Accelerator (ISAC) facility [44] by impinging a $480 \mathrm{MeV}$ proton beam with a current of 25 to $45 \mu \mathrm{A}$ from the TRIUMF cyclotron onto a Ta target. $\mathrm{Yb}$ atoms that left the target were ionized by the TRIUMF resonant ionization laser ion source [45], using a two-step resonant laser excitation scheme into a high-lying Rydberg state $[46,47]$. Ions were extracted and separated using ISAC's high-resolution mass separator [48] at a mass separation power of about 2000. The isobaric beam, consisting mostly of singly charged $\mathrm{Eu}, \mathrm{Dy}, \mathrm{Ho}, \mathrm{Er}, \mathrm{Tm}, \mathrm{Yb}, \mathrm{BaF}$, and $\mathrm{CeO}$ ions, was transported to the TITAN facility, cooled and bunched in the TITAN RF quadrupole cooler buncher [49], and injected into the MR-TOF-MS. There, the ions were transported to the injection trap, cooled, and injected into the TOF analyzer, where they performed one time-focus shift turn [50] and about 330 isochronous turns (IT), corresponding to a TOF of about $8 \mathrm{~ms}$. Then the ions were ejected onto a detector. A mass-resolving power of about 270000 (FWHM) was achieved. For measurements, in which the isobaric contamination was too high to observe $\mathrm{Yb}$ ions, the MR-TOF-MS was first used as an isobar separator: after TOF dispersion of the ions in the analyzer, the ions of interest were retrapped in the injection trap. Then, they were recooled and injected again into the analyzer for the subsequent mass measurement procedure. The overall cycle time was $20 \mathrm{~ms}$. After each mass measurement, a spectrum was taken without resonant laser ionization to verify the identification of the $\mathrm{Yb}$ ions.

Figure 1 shows mass spectra measured without and with retrapping. The mass separation power amounts to 35000 . To avoid deterioration of the mass measurement accuracy due to ion-ion interactions, the beam was attenuated in the ISAC beam line to about one ion per species per cycle detected in the MR-TOF-MS. Using mass-selective retrapping, the rate of contaminant ions was reduced by at least 3 orders of magnitude, and the rate of incoming ions could therefore be increased by a corresponding factor by increasing the proton current on the target and by reducing the attenuation. As shown in Fig. 1, the nuclides ${ }^{151} \mathrm{Tm}$ and ${ }^{151} \mathrm{Yb}$ could only be measured with retrapping. Similarily, the measurement of ${ }^{150} \mathrm{Yb}$ required retrapping. The retrapping increases the dynamic range of the measurement to 5 orders of magnitude, a value which is rarely achieved in 


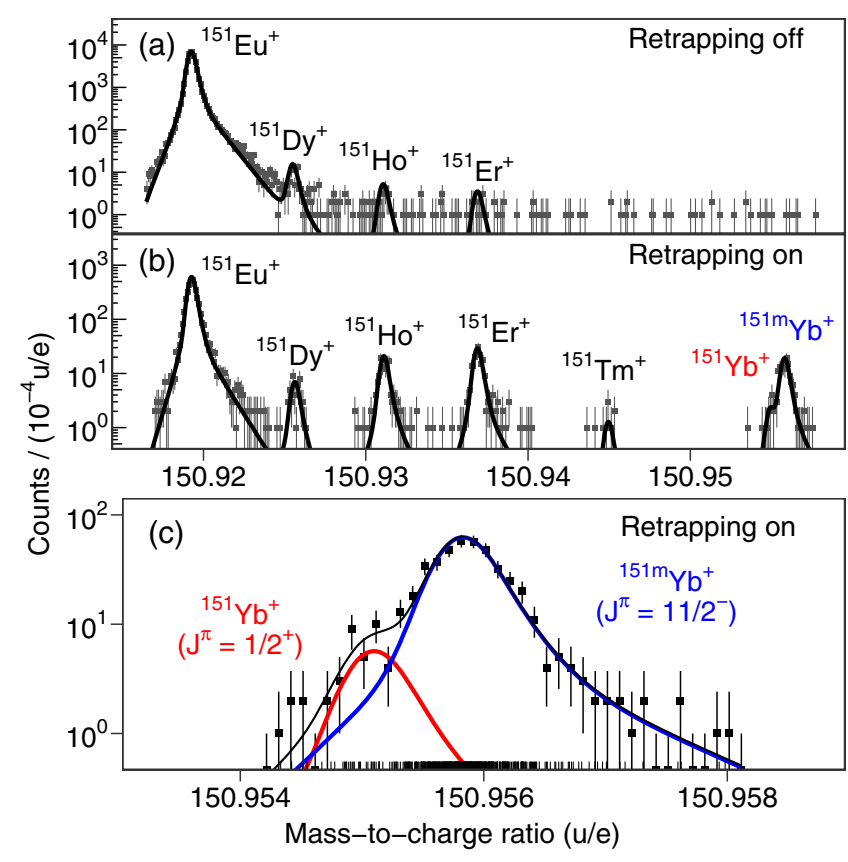

FIG. 1. Mass spectra at mass-to-charge ratio $151 \mathrm{u} / \mathrm{e}$ (a) without retrapping, (b) with retrapping set for ${ }^{151} \mathrm{Yb}$, (c) enlarged to the $\mathrm{Yb}$ region with retrapping, showing the ${ }^{151} \mathrm{Yb}$ nuclear ground and isomeric state. The ions performed 335 IT in the analyzer, corresponding to a TOF of $8.14 \mathrm{~ms}$. The curves represent hyperexponentially modified Gaussian (hyper-EMG) [51] fits to the data. Note the different abundance scales. For both spectra (a) and (b) the measurement time was $760 \mathrm{~s}$; for (b) the incoming rate was increased; (c) contains all data taken during about $3 \mathrm{~h}$ with different proton currents on the target.

mass spectrometry. It also reduces the total number of ions that reach the detector; this minimizes the background resulting from radioactivity implanted on the detector.

For the analysis of the data, the recorded TOF data were converted to mass data using an isobaric ion species present in the mass spectrum to provide a time-resolved calibration [52]. The mass spectra were analyzed by fitting

TABLE I. List of measured mass excess values of $\mathrm{Yb}$ isotopes, $\mathrm{ME}_{\mathrm{TITAN}}$. Mass excess values from the AME2020, $\mathrm{ME}_{\mathrm{AME} 20}$, and the deviation $\Delta \mathrm{ME}=\mathrm{ME}_{\mathrm{TITAN}}-\mathrm{ME}_{\mathrm{AME} 20}$ [53] are given for comparison, where available.

\begin{tabular}{|c|c|c|c|c|}
\hline Nuclide & Calibrant & $\begin{array}{l}\mathrm{ME}_{\text {TITAN }} \\
\left(\mathrm{keV} / c^{2}\right)\end{array}$ & $\begin{array}{l}\mathrm{ME}_{\mathrm{AME} 20} \\
\left(\mathrm{keV} / c^{2}\right)\end{array}$ & $\begin{array}{c}\Delta \mathrm{ME} \\
\left(\mathrm{keV} / c^{2}\right)\end{array}$ \\
\hline${ }^{157} \mathrm{Yb}$ & ${ }^{157} \mathrm{Tm}^{+}$ & $-53395(54)$ & $-53420(11)$ & $25(55)$ \\
\hline${ }^{156} \mathrm{Yb}$ & ${ }^{156} \mathrm{Tm}^{+}$ & $-53331(55)$ & $-53266(9)$ & $-65(56)$ \\
\hline${ }^{155} \mathrm{Yb}$ & ${ }^{155} \mathrm{Eu}^{+}$ & $-50514(45)$ & $-50503(17)$ & $-11(48)$ \\
\hline${ }^{154} \mathrm{Yb}$ & ${ }^{138} \mathrm{Ce}^{16} \mathrm{O}^{+}$ & $-49934(45)$ & $-49932(17)$ & $-2(48)$ \\
\hline${ }^{153} \mathrm{Yb}$ & ${ }^{153} \mathrm{Dy}^{+}$ & $-47102(46)$ & $\ldots$ & \\
\hline${ }^{152} \mathrm{Yb}$ & ${ }^{136} \mathrm{Ce}^{16} \mathrm{O}^{+}$ & $-46061(46)$ & $-46270(150)$ & $209(157)$ \\
\hline${ }^{151} \mathrm{Yb}$ & ${ }^{151} \mathrm{Er}^{+}$ & $-41297(114)$ & $-41540(300)$ & $243(321)$ \\
\hline${ }^{151} \mathrm{Yb}^{\mathrm{m}}$ & ${ }^{151} \mathrm{Er}^{+}$ & $-40617(49)$ & $\ldots$ & \\
\hline${ }^{150} \mathrm{Yb}$ & ${ }^{150} \mathrm{Dy}^{+}$ & $-38635(44)$ & $\ldots$ & \\
\hline
\end{tabular}

hyper-EMG functions [51] to the unbinned mass data using weighted maximum likelihood estimation [52]. The isotopes of interest and their respective calibrants are listed in Table I. The mass values of the calibrants were taken from the atomic mass evaluation (AME2020) [53]. The dominating contribution to the systematic uncertainty are shifts in the TOF due to voltage ringing caused by the switching of the reflector voltages $[54,55]$. Its relative value amounts to $3 \times 10^{-7}$.

The ground state masses of eight $\mathrm{Yb}$ isotopes were measured (Table I). For ${ }^{154-157} \mathrm{Yb}$, the masses were already well known, and the present results are in good agreement with the AME2020 [53]. The masses of ${ }^{151} \mathrm{Yb}$ and ${ }^{152} \mathrm{Yb}$ were measured directly for the first time, and their uncertainties could be reduced by a factor of 3 . The masses of ${ }^{150} \mathrm{Yb}$ and ${ }^{153} \mathrm{Yb}$ were measured for the first time.

With these results, the $N=82$ shell closure can be examined in the extreme proton-rich region. Figure 2 shows the empirical two-neutron-shell gap $\Delta_{2 n}(Z, N)=$ $S_{2 n}(Z, N)-S_{2 n}(Z, N+2)$, where $S_{2 n}(Z, N)=B(Z, N)-$ $B(Z, N-2)$ is the two-neutron separation energy and $B(Z, N)$ is the binding energy, for different $N=82$ isotones. The two-neutron-shell gap shows pronounced maxima when crossing closed shells. From the proton shell closure at $Z=50$, the two-neutron-shell gap decreases, though from $Z=58$ onward, the reduction is only weak. So far, the most proton-rich nuclide, for which $\Delta_{2 n}$ was known, was ${ }^{150} \operatorname{Er}(Z=68)$. The newly determined value for ${ }^{152} \mathrm{Yb}(Z=70)$, despite being the lowest value found so far, clearly establishes that the shell persists with almost unmodified shell gap energy up to the proton drip line. The drip line is expected to lie between ${ }^{152} \mathrm{Yb}$ and ${ }^{153} \mathrm{Lu}$ $(Z=71)$ [53,56]. The experimental data are compared with different theoretical models, the macroscopic-microscopic finite-range droplet model FRDM(2012) [57], and two microscopic models, the Hartree-Fock-Bogoliubov

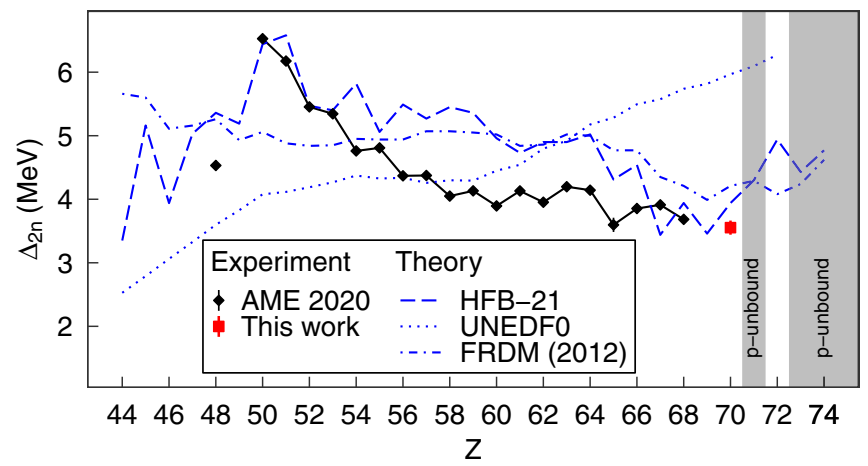

FIG. 2. Evolution of the two-neutron-shell gap $\Delta_{2 n}$ at $N=82$ as a function of the proton number $Z$ and predictions of the theoretical models HFB21 [58], UNEDF0 [59], and FRDM2012 [57]. Experimental data are from the AME2020 [53] and this Letter. Regions of proton-unbound nuclides are indicated [53,56]. Most error bars are hidden in the symbols; lines are drawn to guide the eye. 
model with BSk21 Skyrme interaction (HFB-21) [58], and the energy density functional UNEDF0 [59]. Although HFB-21 comes closest to the measured values, in particular for the most proton-rich isotones, none of these models fully reproduces the experimental trend. This fact highlights the importance of measurements for model improvements and model error estimates.

Furthermore, the mass of ${ }^{153} \mathrm{Yb}$ provides an anchor point for the $\alpha$ decay chains from ${ }^{173} \mathrm{Hg}$ to ${ }^{153} \mathrm{Yb}$ and from ${ }^{170} \mathrm{Au}$ to ${ }^{154} \mathrm{Lu}$ and thus determines the absolute masses of nine more nuclides and fixes the mass surface in this region of the chart of nuclides $[60,61]$.

A $J^{\pi}=11 / 2^{-}$isomer has been observed in ${ }^{151} \mathrm{Yb}$ previously [62-64], but in this work its excitation energy was measured for the first time [Fig. 1(c)]. In total, 460 events were detected with an isomer-to-ground state ratio of 11.1(3.1), corresponding to 38 events in the ground state. The measured excitation energy is $679(105) \mathrm{keV}$; it falls in line with the excitation energy of about $750 \mathrm{keV}$ of $J^{\pi}=11 / 2^{-}$isomers in the even $Z, N=81$ isotones from ${ }^{139} \mathrm{Ce}$ onward. The experimental data are shown in Fig. 3(a). The systematic trend suggests the assignment of the measured $\mathrm{Yb}$ isomer as $J^{\pi}=11 / 2^{-}$[25,63]. The $J^{\pi}=1 / 2^{+}, 3 / 2^{+}$, and $11 / 2^{-}$states are neutron-hole states below the closed shell and can be associated with the $s_{1 / 2}$, $d_{3 / 2}$, and $h_{11 / 2}$ orbitals, respectively. The fact that the excitation energies are constant has not been explained so far $[22,25,26]$.

In order to resolve this long-standing riddle, mean-field calculations were performed. The phenomenological, deformed Woods-Saxon Hamiltonian in its so-called universal parametrization, for which its parameters are

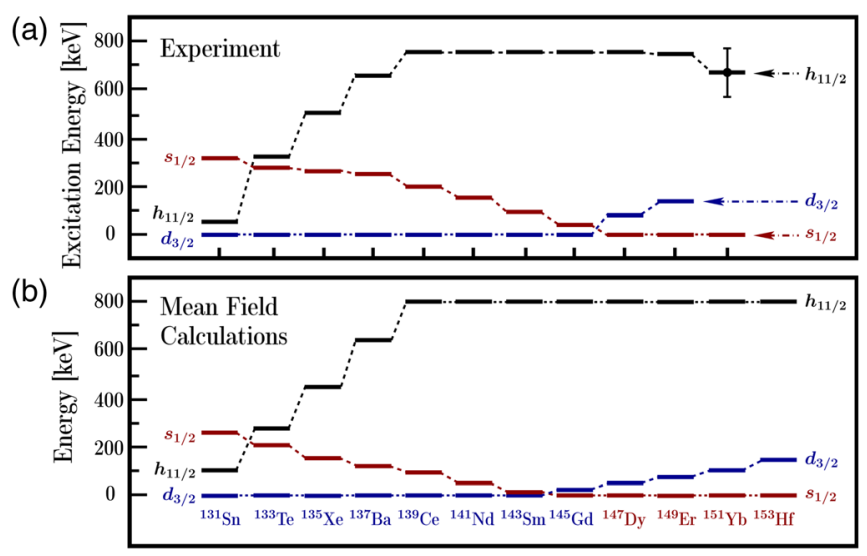

FIG. 3. (a) Measured excitation energies of isomers in the even$Z N=81$ isotones from $\mathrm{Sn}$ to $\mathrm{Yb}[19,25]$. The value for ${ }^{151} \mathrm{Yb}$ results from the present work. Most error bars are invisible within the scale of the figure. Note the constancy of the $h_{11 / 2}$ excitation energy from $\mathrm{Ce}$ to $\mathrm{Yb}$. (b) Corresponding results obtained using mean-field calculations with universal parametrization of the Woods-Saxon Hamiltonian. From $\mathrm{Nd}(Z=60)$ to $\mathrm{Hf}(Z=72)$, the filled proton levels are near-degenerate, cf. Fig. 4. fixed throughout the chart of nuclides, was employed [65-69]. Its use is supported by the fact that it has been successfully applied in numerous nuclear structure calculations. Furthermore, it has recently been tested extensively from the point of view of prediction uncertainties and elimination of parametric correlations [70], which are known to destroy-often completely-model prediction capacities [71].

Potential-energy calculations using the Strutinsky method [72] were performed for all even- $Z$ isotones from Sn to Hf. Partial results are shown in Fig. 3(b). The $J^{\pi}=$ $3 / 2^{+}$ground states with even $Z$ from $\mathrm{Sn}$ to $\mathrm{Gd}$ can be associated with $d_{3 / 2}$, and $J^{\pi}=1 / 2^{+}$with $s_{1 / 2}$ orbitals from Dy to Hf. The latter orbital does not couple with the spinorbit field at all, whereas the former does so only very weakly, so that their crossing at Gd and Dy reflects mainly the evolution of the central potential with $Z$.

Calculations show the impact of the shell closures at $Z=50$ and $N=82$, in that the ground-state equilibrium shapes remain spherical for the $\mathrm{Sn}, \mathrm{Te}, \mathrm{Xe}$, and $\mathrm{Ba}$ isotones, though the potential stiffness decreases. They predict nonspherical (oblate) quadrupole equilibrium shapes for ${ }^{139} \mathrm{Ce}$ and heavier isotones, while predicting small but increasingly prolate shapes for the $J^{\pi}=11 / 2^{-}$isomers. This evolution coincides with the evolution of the energies of the isomers, which increase from about $40 \mathrm{keV}$ in ${ }^{131} \mathrm{Sn}$ to about $750 \mathrm{keV}$ in ${ }^{139} \mathrm{Ce}$, i.e., within the zone of spherical ground states. Stabilization at about $750 \mathrm{keV}$, starting with $\mathrm{Ce}(Z=58)$, coincides with the mean-field predictions of the slightly prolate quadrupole shapes with quadrupole deformations of $\alpha_{20} \approx 0.10$ at the $J^{\pi}=11 / 2^{-}$isomeric energy minima. The calculations also show that the trend of constant $J^{\pi}=11 / 2^{-}$isomer excitation energies continues for ${ }^{153} \mathrm{Hf}$.

Below spherical closed neutron shells (here $N=82$ ), $K$ isomers usually correspond to nucleonic configurations with maximum alignment of the angular momentum $j$, i.e., with projection $m_{j}=j$, at slightly prolate quadrupole shapes [27]. Calculations suggest that $h_{11 / 2}$ isomers have

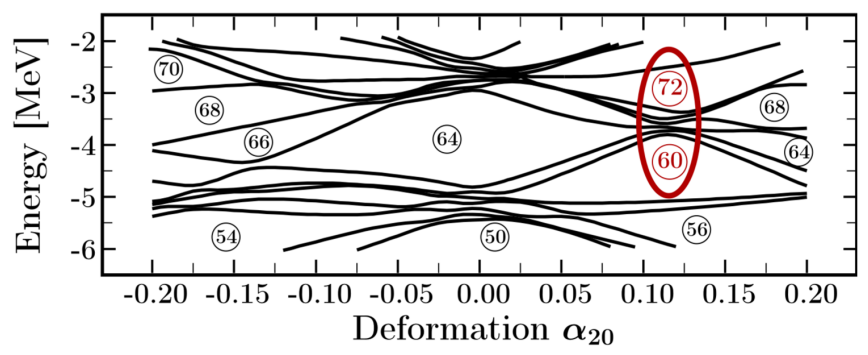

FIG. 4. Single proton energies as functions of the quadrupole deformation $\alpha_{20}$, calculated using the Woods-Saxon Hamiltonian. The numbers in the circles represent the number of protons that can fill the levels below the circles. Note a near-degeneracy of six levels at small prolate deformations, indicated with oval curve, cf. Nd-Hf evolution in Fig. 3. 
typical prolate deformations of $\alpha_{20} \approx 0.1$. The underlying stabilizing structural element is the very high singlenucleonic (proton) density at $\alpha_{20} \approx 0.12$ (Fig. 4), where six proton levels lie very close together. According to the Strutinsky shell-correction approach, the corresponding shell energies are strongly negative and nearly constant, stabilizing the corresponding shapes with isomer energies at about $800 \mathrm{keV}$.

In summary, high-accuracy mass measurements of neutron-deficient $\mathrm{Yb}$ isotopes have been performed. They were enabled by the first-ever simultaneous on-line use of an MR-TOF-MS as an isobar separator and as a mass spectrometer, employing mass-selective retrapping, and thus extending the measurements to two isotopes further away from stability than otherwise possible. The persistence of the $N=82$ shell with almost unmodified shell gap energies was established up to the expected location of the proton drip line. Furthermore, the results extend the knowledge of excitation energies of the unique $J^{\pi}=11 / 2^{-}$ isomers in even- $Z, N=81$ isotones. The structural properties of this sequence were analyzed, and the constant excitation energies over a range of 13 isotones was explained. Application of the mass-selective retrapping is not limited to TITAN, but could also be employed with other MR-TOF-MS worldwide to extend the reach of mass measurements with these devices by two or more isotopes toward exoticity. Since its first use in this experiment, this technique is now regularly applied in TITAN's MR-TOF-MS.

We would like to thank J. Lassen and the TRILIS ion source group for the excellent beam conditions. This work was supported by the German Research Foundation (DFG), Grant No. SCHE 1969/2-1; by the German Federal Ministry for Education and Research (BMBF), Grants No. 05P16RGFN1 and No. 05P19RGFN1; by the Hessian Ministry for Science and Art through the LOEWE Center HIC for FAIR; by the JLU and GSI Helmholtzzentrum für Schwerionenforschung under the JLU-GSI strategic Helmholtz partnership agreement; by the Polish National Science Centre under Contract No. 2016/21/B/ST2/01227; and by the UKRI Science and Technology Facilities Council (STFC), Grant No. ST/P004008/1. Support from the National Natural Science Foundation of China, Grant No. 11975209, and the Physics Research and Development Program of Zhengzhou University, Grant No. 32410017, are acknowledged. TITAN is funded by the Natural Sciences and Engineering Research Council (NSERC) of Canada and through TRIUMF by the National Research Council (NRC) of Canada. N. M. acknowledges the support by the FAIR Phase-0 project and by Bulgarian NSF Contract No. KP06-N48/1. E. D. acknowledges the support by the Canada-UK Foundation.
* Corresponding author. s.beck@gsi.de

[1] K. Riisager, Nuclear halo states, Rev. Mod. Phys. 66, 1105 (1994).

[2] M. Pfützner, M. Karny, L. V. Grigorenko, and K. Riisager, Radioactive decays at limits of nuclear stability, Rev. Mod. Phys. 84, 567 (2012).

[3] I. Dedes and J. Dudek, Propagation of the nuclear meanfield uncertainties with increasing distance from the parameter adjustment zone: Applications to superheavy nuclei, Phys. Rev. C 99, 054310 (2019).

[4] O. Sorlin and M.-G. Porquet, Nuclear magic numbers: New features far from stability, Prog. Part. Nucl. Phys. 61, 602 (2008).

[5] R. Kanungo, A new view of nuclear shells, Phys. Scr. T152, 014002 (2013).

[6] C. Thibault, R. Klapisch, C. Rigaud, A. M. Poskanzer, R. Prieels, L. Lessard, and W. Reisdorf, Direct measurement of the masses of ${ }^{11} \mathrm{Li}$ and ${ }^{26-32} \mathrm{Na}$ with an on-line mass spectrometer, Phys. Rev. C 12, 644 (1975).

[7] F. Sarazin, H. Savajols, W. Mittig, F. Nowacki, N. A. Orr, Z. Ren, P. Roussel-Chomaz, G. Auger, D. Baiborodin, A. V. Belozyorov et al., Shape Coexistence and the $N=28$ Shell Closure Far from Stability, Phys. Rev. Lett. 84, 5062 (2000).

[8] A. T. Gallant, J. C. Bale, T. Brunner, U. Chowdhury, S. Ettenauer, A. Lennarz, D. Robertson, V. V. Simon, A. Chaudhuri, J. D. Holt et al., New Precision Mass Measurements of Neutron-Rich Calcium and Potassium Isotopes and Three-Nucleon Forces, Phys. Rev. Lett. 109, 032506 (2012).

[9] F. Wienholtz, D. Beck, K. Blaum, C. Borgmann, M. Breitenfeldt, R. B. Cakirli, S. George, F. Herfurth, J. D. Holt, M. Kowalska et al., Masses of exotic calcium isotopes pin down nuclear forces, Nature (London) 498, 346 (2013).

[10] M. Rosenbusch, P. Ascher, D. Atanasov, C. Barbieri, D. Beck, K. Blaum, C. Borgmann, M. Breitenfeldt, R. B. Cakirli, A. Cipollone et al., Probing the $N=32$ Shell Closure below the Magic Proton Number $Z=20$ : Mass Measurements of the Exotic Isotopes ${ }^{52,53} \mathrm{~K}$, Phys. Rev. Lett. 114, 202501 (2015).

[11] X. Xu, M. Wang, Y.-H. Zhang, H.-S. Xu, P. Shuai, X.-L. Tu, Y. A. Litvinov, X.-H. Zhou, B.-H. Sun, Y.-J. Yuan et al., Direct mass measurements of neutron-rich ${ }^{86} \mathrm{Kr}$ projectile fragments and the persistence of neutron magic number $N=32$ in Sc isotopes, Chin. Phys. C 39, 104001 (2015).

[12] E. Leistenschneider, M. P. Reiter, S. Ayet San Andrés, B. Kootte, J. D. Holt, P. Navrátil, C. Babcock, C. Barbieri, B. R. Barquest, J. Bergmann et al., Dawning of the $N=32$ Shell Closure Seen through Precision Mass Measurements of Neutron-Rich Titanium Isotopes, Phys. Rev. Lett. 120, 062503 (2018).

[13] D. Steppenbeck, S. Takeuchi, N. Aoi, P. Doornenbal, M. Matsushita, H. Wang, H. Baba, N. Fukuda, S. Go, M. Honma et al., Evidence for a new nuclear 'magic number' from the level structure of ${ }^{54} \mathrm{Ca}$, Nature (London) 502, 207 (2013).

[14] S. Michimasa, M. Kobayashi, Y. Kiyokawa, S. Ota, D. S. Ahn, H. Baba, G. P. A. Berg, M. Dozono, N. Fukuda, 
T. Furuno et al., Magic Nature of Neutrons in ${ }^{54} \mathrm{Ca}$ : First Mass Measurements of ${ }^{55-57} \mathrm{Ca}$, Phys. Rev. Lett. 121, 022506 (2018).

[15] E. Leistenschneider, E. Dunling, G. Bollen, B. A. Brown, J. Dilling, A. Hamaker, J. D. Holt, A. Jacobs, A. A. Kwiatkowski, T. Miyagi et al. (The LEBIT and the TITAN Collaborations), Precision Mass Measurements of NeutronRich Scandium Isotopes Refine the Evolution of $N=32$ and $N=34$ Shell Closures, Phys. Rev. Lett. 126, 042501 (2021).

[16] D. Atanasov, P. Ascher, K. Blaum, R. B. Cakirli, T.E. Cocolios, S. George, S. Goriely, F. Herfurth, H.-T. Janka, O. Just et al., Precision Mass Measurements of ${ }^{129-131} \mathrm{Cd}$ and Their Impact on Stellar Nucleosynthesis via the Rapid Neutron Capture Process, Phys. Rev. Lett. 115, 232501 (2015).

[17] R. Knöbel, M. Diwisch, F. Bosch, D. Boutin, L. Chen, C. Dimopoulou, A. Dolinskii, B. Franczak, B. Franzke, H. Geissel et al., First direct mass measurements of stored neutron-rich ${ }^{129,130,131} \mathrm{Cd}$ isotopes with FRS-ESR, Phys. Lett. B 754, 288 (2016).

[18] V. Manea, J. Karthein, D. Atanasov, M. Bender, K. Blaum, T. E. Cocolios, S. Eliseev, A. Herlert, J. D. Holt, W. J. Huang et al., First Glimpse of the $N=82$ Shell Closure below $Z=50$ from Masses of Neutron-Rich Cadmium Isotopes and Isomers, Phys. Rev. Lett. 124, 092502 (2020).

[19] F. Kondev, M. Wang, W. Huang, S. Naimi, and G. Audi, The NUBASE2020 evaluation of nuclear physics properties, Chin. Phys. C 45, 030001 (2021).

[20] From ENSDF database as of October, 2020, https://www .nndc.bnl.gov/ensarchivals/.

[21] B. H. Ketelle, H. Thomas, and A. R. Brosi, Decay of the Cerium-139 isomers, Phys. Rev. 103, 190 (1956).

[22] K. Kotajima and H. Morinaga, Isomers in $N=81$ nuclei, Nucl. Phys. 16, 231 (1960).

[23] G. Jansen, H. Morinaga, and C. Signorini, A new isomeric state in $N=81$ nuclei: ${ }^{145 \mathrm{~m}} \mathrm{Gd}$, Nucl. Phys. A128, 247 (1969).

[24] K. Toth, A. Rainis, C. Bingham, E. Newman, H. Carter, and W.-D. Schmidt-Ott, Excitation energies of the $\mathrm{h}_{11 / 2}$ and $\mathrm{d}_{3 / 2}$ neutron states in ${ }^{145} \mathrm{Gd}$ and ${ }^{147} \mathrm{Dy}$, Phys. Lett. 56B, 29 (1975).

[25] K. S. Toth, Y. A. Ellis-Akovali, F. T. Avignone, R. S. Moore, D. M. Moltz, J. M. Nitschke, P. A. Wilmarth, P. K. Lemmertz, D. C. Sousa, and A. L. Goodman, Single-particle states in ${ }^{149} \mathrm{Er}$ and ${ }^{149} \mathrm{Ho}$, and the effect of the $Z=64$ closure, Phys. Rev. C 32, 342 (1985).

[26] K. Heyde and P. J. Brussaard, Neutron hole states in the $N=81$ nuclei, Z. Phys. A 259, 15 (1973).

[27] M. J. A. de Voigt, J. Dudek, and Z. Szymanski, High-spin phenomena in atomic nuclei, Rev. Mod. Phys. 55, 949 (1983).

[28] H. Wollnik and M. Przewloka, Time-of-flight mass spectrometers with multiply reflected ion trajectories, Int. J. Mass Spectrom. Ion Process. 96, 267 (1990).

[29] W. R. Plaß, T. Dickel, and C. Scheidenberger, Multiplereflection time-of-flight mass spectrometry, Int. J. Mass Spectrom. 349-350, 134 (2013).

[30] W. R. Plaß, T. Dickel, U. Czok, H. Geissel, M. Petrick, K. Reinheimer, C. Scheidenberger, and M. I. Yavor, Isobar separation by time-of-flight mass spectrometry for low-energy radioactive ion beam facilities, Nucl. Instrum. Methods Phys. Res., Sect. B 266, 4560 (2008).

[31] R. N. Wolf, D. Beck, K. Blaum, C. Böhm, C. Borgmann, M. Breitenfeldt, N. Chamel, S. Goriely, F. Herfurth, M. Kowalska et al., Plumbing Neutron Stars to New Depths with the Binding Energy of the Exotic Nuclide ${ }^{82} \mathrm{Zn}$, Phys. Rev. Lett. 110, 041101 (2013).

[32] T. Dickel, W. R. Plaß, S. Ayet San Andrés, J. Ebert, H. Geissel, E. Haettner, C. Hornung, I. Miskun, S. Pietri, S. Purushothaman et al., First spatial separation of a heavy ion isomeric beam with a multiple-reflection time-of-flight mass spectrometer, Phys. Lett. B 744, 137 (2015).

[33] Y. Ito, P. Schury, M. Wada, F. Arai, H. Haba, Y. Hirayama, S. Ishizawa, D. Kaji, S. Kimura, H. Koura et al., First Direct Mass Measurements of Nuclides around $Z=100$ with a Multireflection Time-of-Flight Mass Spectrograph, Phys. Rev. Lett. 120, 152501 (2018).

[34] C. Hornung, D. Amanbayev, I. Dedes, G. Kripko-Koncz, I. Miskun, N. Shimizu, S. Ayet San Andrés, J. Bergmann, T. Dickel, J. Dudek et al., Isomer studies in the vicinity of the doubly-magic nucleus ${ }^{100} \mathrm{Sn}$ : Observation of a new lowlying isomeric state in ${ }^{97} \mathrm{Ag}$, Phys. Lett. B 802, 135200 (2020).

[35] S. Purushothaman, M. P. Reiter, E. Haettner, P. Dendooven, T. Dickel, H. Geissel, J. Ebert, C. Jesch, W. R. Plaß, M. Ranjan et al., First experimental results of a cryogenic stopping cell with short-lived, heavy uranium fragments produced at $1000 \mathrm{MeV} / \mathrm{u}$, Europhys. Lett. 104, 42001 (2013).

[36] R. Wolf, F. Wienholtz, D. Atanasov, D. Beck, K. Blaum, C. Borgmann, F. Herfurth, M. Kowalska, S. Kreim, Y. A. Litvinov et al., ISOLTRAP's multi-reflection time-of-flight mass separator/spectrometer, Int. J. Mass Spectrom. 349350, 123 (2013).

[37] M. P. Reiter, F. Ames, C. Andreoiu, S. Ayet San Andrés, C. Babcock, B. R. Barquest, J. Bergmann, J. Bollig, T. Brunner, T. Dickel et al., Improved beam diagnostics and optimization at ISAC via TITAN's MR-TOF-MS, Nucl. Instrum. Methods Phys. Res., Sect. B 463, 431 (2020).

[38] F. Wienholtz, S. Kreim, M. Rosenbusch, L. Schweikhard, and R. Wolf, Mass-selective ion ejection from multireflection time-of-flight devices via a pulsed in-trap lift, Int. J. Mass Spectrom. 421, 285 (2017).

[39] J. T. Johnson, I. J. Carrick, G. S. Eakins, and S. A. McLuckey, Mirror switching for high-resolution ion isolation in an electrostatic linear ion trap, Anal. Chem. 91, 8789 (2019).

[40] T. Dickel, W. R. Plaß, W. Lippert, J. Lang, M. I. Yavor, H. Geissel, and C. Scheidenberger, Isobar separation in a multiple-reflection time-of-flight mass spectrometer by mass-selective re-trapping, J. Am. Soc. Mass Spectrom. 28, 1079 (2017).

[41] C. Jesch, T. Dickel, W. R. Plaß, D. Short, S. Ayet San Andres, J. Dilling, H. Geissel, F. Greiner, J. Lang, K. G. Leach et al., The MR-TOF-MS isobar separator for the TITAN facility at TRIUMF, Hyperfine Interact. 235, 97 (2015).

[42] T. Dickel, S. Ayet San Andres, S. Beck, J. Bergmann, J. Dilling, F. Greiner, C. Hornung, A. Jacobs, 
G. Kripko-Koncz, A. Kwiatkowski et al., Recent upgrades of the multiple-reflection time-of-flight mass spectrometer at TITAN, Hyperfine Interact. 240, 62 (2019).

[43] J. Dilling, R. Baartman, P. Bricault, M. Brodeur, L. Blomeley, F. Buchinger, J. Crawford, J. R. C. LopezUrrutia, P. Delheij, M. Froese et al., Mass measurements on highly charged radioactive ions, a new approach to high precision with TITAN, Int. J. Mass Spectrom. 251, 198 (2006).

[44] M. Dombsky, D. Bishop, P. Bricault, D. Dale, A. Hurst, K. Jayamanna, R. Keitel, M. Olivo, P. Schmor, and G. Stanford, Commissioning and initial operation of a radioactive beam ion source at ISAC, Rev. Sci. Instrum. 71, 978 (2000).

[45] S. Raeder, H. Heggen, J. Lassen, F. Ames, D. Bishop, P. Bricault, P. Kunz, A. Mjøs, and A. Teigelhöfer, An ion guide laser ion source for isobar-suppressed rare isotope beams, Rev. Sci. Instrum. 85, 033309 (2014).

[46] J. Lassen, R. Li, S. Raeder, X. Zhao, T. Dekker, H. Heggen, P. Kunz, C. D. P. Levy, M. Mostanmand, A. Teigelhöfer, and F. Ames, Current developments with TRIUMF's titaniumsapphire laser based resonance ionization laser ion source, Hyperfine Interact. 238, 33 (2017).

[47] H. Backe, P. Kunz, W. Lauth, A. Dretzke, R. Horn, T. Kolb, M. Laatiaoui, M. Sewtz, D. Ackermann, M. Block et al., Towards optical spectroscopy of the element nobelium $(Z=102)$ in a buffer gas cell, Eur. Phys. J. D 45, 99 (2007).

[48] P. Bricault, R. Baartman, M. Dombsky, A. Hurst, C. Mark, G. Stanford, and P. Schmor, TRIUMF-ISAC target station and mass separator commissioning, Nucl. Phys. A701, 49 (2002).

[49] T. Brunner, M. J. Smith, M. Brodeur, S. Ettenauer, A. T. Gallant, V. V. Simon, A. Chaudhuri, A. Lapierre, E. Mane, R. Ringle et al., TITAN's digital RFQ ion beam cooler and buncher, operation and performance, Nucl. Instrum. Methods Phys. Res., Sect. A 676, 32 (2012).

[50] T. Dickel, M. I. Yavor, J. Lang, W. R. Plaß, W. Lippert, H. Geissel, and C. Scheidenberger, Dynamical time focus shift in multiple-reflection time-of-flight mass spectrometers, Int. J. Mass Spectrom. 412, 1 (2017).

[51] S. Purushothaman, S. Ayet San Andrés, J. Bergmann, T. Dickel, J. Ebert, H. Geissel, C. Hornung, W. R. Plaß, C. Rappold, C. Scheidenberger et al., Hyper-EMG: A new probability distribution function composed of Exponentially Modified Gaussian distributions to analyze asymmetric peak shapes in high-resolution time-of-flight mass spectrometry, Int. J. Mass Spectrom. 421, 245 (2017).

[52] S. Ayet San Andrés, C. Hornung, J. Ebert, W. R. Plaß, T. Dickel, H. Geissel, C. Scheidenberger, J. Bergmann, F. Greiner, E. Haettner et al., High-resolution, accurate multiple-reflection time-of-flight mass spectrometry for short-lived, exotic nuclei of a few events in their ground and low-lying isomeric states, Phys. Rev. C 99, 064313 (2019).

[53] M. Wang, W. Huang, F. Kondev, G. Audi, and S. Naimi, The AME 2020 atomic mass evaluation (II). Tables, graphs and references, Chin. Phys. C 45, 030003 (2021).

[54] C. Will, TITAN's multiple-reflection time-of-flight mass spectrometer and isobar separator-characterization and first experiments, Bachelor thesis, Justus Liebig University Gießen, 2017.
[55] M. P. Reiter, S. Ayet San Andrés, E. Dunling, B. Kootte, E. Leistenschneider, C. Andreoiu, C. Babcock, B. R. Barquest, J. Bollig, T. Brunner et al., Quenching of the $N=32$ neutron shell closure studied via precision mass measurements of neutron-rich vanadium isotopes, Phys. Rev. C 98, 024310 (2018).

[56] L. Neufcourt, Y. Cao, S. Giuliani, W. Nazarewicz, E. Olsen, and O. B. Tarasov, Beyond the proton drip line: Bayesian analysis of proton-emitting nuclei, Phys. Rev. C 101, 014319 (2020).

[57] P. Möller, A. J. Sierk, T. Ichikawa, and H. Sagawa, Nuclear ground-state masses and deformations: FRDM(2012), At. Data Nucl. Data Tables 109-110, 1 (2016).

[58] S. Goriely, N. Chamel, and J. M. Pearson, Further explorations of Skyrme-Hartree-Fock-Bogoliubov mass formulas. XII. Stiffness and stability of neutron-star matter, Phys. Rev. C 82, 035804 (2010).

[59] M. Kortelainen, T. Lesinski, J. Moré, W. Nazarewicz, J. Sarich, N. Schunck, M. V. Stoitsov, and S. Wild, Nuclear energy density optimization, Phys. Rev. C 82, 024313 (2010).

[60] M. Wang, G. Audi, F. G. Kondev, W. J. Huang, S. Naimi, and $\mathrm{X}$. $\mathrm{Xu}$, The AME2016 atomic mass evaluation (II). Tables, graphs and references, Chin. Phys. C 41, 030003 (2017).

[61] E. M. Lykiardopoulou et al. (to be published).

[62] P. Kleinheinz, B. Rubio, M. Ogawa, M. Piiparinen, A. Plochocki, D. Schardt, R. Barden, O. Klepper, R. Kirchner, and E. Roeckl, Beta-decay of ${ }^{151} \mathrm{Yb}$, Z. Phys. A 322, 705 (1985).

[63] K. Toth, Y. Ellis-Akovali, J. Nitschke, P. Wilmarth, P. Lemmertz, D. Moltz, and F. Avignone, Structure in $\beta$ delayed proton spectra of $N=81$ precursors, Phys. Lett. B 178, 150 (1986).

[64] Y. A. Akovali, K. S. Toth, A. L. Goodman, J. M. Nitschke, P. A. Wilmarth, D. M. Moltz, M. N. Rao, and D. C. Sousa, Single-particle states in ${ }^{151} \mathrm{Tm}$ and ${ }^{151} \mathrm{Er}$ : Systematics of neutron states in $N=83$ nuclei, Phys. Rev. C 41, 1126 (1990).

[65] J. Dudek and T. Werner, New parameters of the deformed Woods-Saxon potential for $A=110-210$ nuclei, J. Phys. G 4, 1543 (1978).

[66] J. Dudek, A. Majhofer, J. Skalski, T. Werner, S. Cwiok, and W. Nazarewicz, Parameters of the deformed Woods-Saxon potential outside $A=110-210$ nuclei, J. Phys. G 5, 1359 (1979).

[67] J. Dudek, W. Nazarewicz, and T. Werner, Discussion of the improved parametrisation of the Woods-Saxon potential for deformed nuclei, Nucl. Phys. A341, 253 (1980).

[68] J. Dudek, Z. Szymański, and T. Werner, Woods-Saxon potential parameters optimized to the high spin spectra in the lead region, Phys. Rev. C 23, 920 (1981).

[69] S. Cwiok, J. Dudek, W. Nazarewicz, J. Skalski, and T. Werner, Single-particle energies, wave functions, quadrupole moments and $g$-factors in an axially deformed woodssaxon potential with applications to the two-centre-type nuclear problems, Comput. Phys. Commun. 46, 379 (1987).

[70] A. Gaamouci, I. Dedes, J. Dudek, A. Baran, N. Benhamouda, D. Curien, H. L. Wang, and J. Yang, Exotic toroidal and superdeformed configurations in light 
atomic nuclei: Predictions using a mean-field Hamiltonian without parametric correlations, Phys. Rev. C 103, 054311 (2021).

[71] I. Dedes and J. Dudek, Predictive power of theoretical modelling of the nuclear mean field: examples of improving predictive capacities, Phys. Scr. 93, 044003 (2018).

[72] V. Strutinsky, Shell effects in nuclear masses and deformation energies, Nucl. Phys. A95, 420 (1967). 